\title{
Treatment of COVID-19 atypical pneumonia by early Tocilizumab administration in "non-critically-ill" patients on hemodialysis
}

\author{
Giuseppe Castellano $^{1}$ - Barbara Infante ${ }^{1}$ Silvia Mercuri ${ }^{1}$. Mauro Forcella ${ }^{1}$ Sharon Natasha Cox ${ }^{3}$. \\ Gaetano Serviddio $^{2} \cdot$ Loreto Gesualdo $^{3} \cdot$ Giovanni Stallone $^{1}$
}

Received: 9 May 2020 / Accepted: 9 September 2020 / Published online: 30 September 2020

(c) Italian Society of Nephrology 2020

Keywords Tocilizumab · Dialysis $\cdot$ IL-6 $\cdot$ COVID-19

\section{The cases}

Patient 1. A 49-year-old woman on hemodialysis (HD) because of IgA nephropathy was admitted with a dry cough and fever that persisted for $48 \mathrm{~h}\left(38.5^{\circ} \mathrm{C}\right)$, without dyspnea; she was found positive to SARS-CoV-2 (RT-PCR assay). She had been on HD since 1996, received a kidney transplant in 2007, which failed in 2018. On examination O2 saturation was $92 \%$ in room air, arterial $\mathrm{O} 2$ tension $(\mathrm{PaO} 2)$ was $68 \mathrm{mmHg}$. Chest X-Ray showed interstitial thickening in the right lower perihilar area and a small parenchymal consolidation in the left perihilar area. She was treated with hydroxychloroquine ( $200 \mathrm{mg}$ two times/day for two days, then $200 \mathrm{mg} /$ post-HD for ten days), ceftriaxone ( $1 \mathrm{~g} /$ day), $\mathrm{N}$-acetyl cysteine (300 $\mathrm{mg}$ two times/day), prednisone $(5 \mathrm{mg} /$ day), enoxaparin (8000-10,000 UI/day) and oxygen therapy at an initial dose of $6 \mathrm{lit} / \mathrm{min}$. However, we found a progressive alteration of biomarkers, including low lymphocyte and monocyte counts, increased C-reactive protein (CRP), high LDH levels [1] (Fig. 1a) and increased requirement of oxygen therapy (rising up to $8-10 \mathrm{lit} / \mathrm{min}$ ); chest

Giuseppe Castellano and Barbara Infante equally contributed to the present study.

Giuseppe Castellano

giuseppe.castellano@unifg.it

1 Department of Medical and Surgical Sciences, Nephrology, Dialysis and Transplantation Unit, University of Foggia, Viale Luigi Pinto, 1, 71100 Foggia, Italy

2 Liver Unit, Centro Universitario Per La Ricerca E La Cura Delle Epatopatie (C.U.R.E.), Department of Medical and Surgical Sciences, University of Foggia, Foggia, Italy

3 Nephrology, Dialysis and Transplantation Unit, Department of Emergency and Organ Transplantation, University of Bari "Aldo Moro", Bari, Italy high-resolution computed tomography (HRCT) confirmed atypical pneumonia involving over $60 \%$ of the lung parenchyma (Fig. 1b, c). Six days later she showed no improvement and a significant increase in IL-6 levels $(41.07 \mathrm{pg} / \mathrm{ml}$, normal range $0.5-6.4)$. Tocilizumab rescue therapy was started $(8 \mathrm{mg} / \mathrm{kg})$. One day later cough and fever attenuated and we observed a progressive normalization of lymphocyte and monocyte counts as well as of CRP and LDH levels (Fig. 1a), associated with complete disappearance of lung lesions (Fig. 1d-f). Interestingly, the oropharyngeal swab test became negative only after 37 days.

Patient 2. A 36-year-old woman on HD regimen for six years was admitted to our unit with fever $\left(37.2^{\circ} \mathrm{C}\right)$, and a dry cough that started 3 days before admission. Initial evaluation showed $\mathrm{O} 2$ saturation $88 \%$ in room air, $\mathrm{PaO} 2$ of $52 \mathrm{mmHg}$ and positivity to SARS-CoV-2 swab. After observing a significant decrease in lymphocyte and monocyte counts, and altered CRP and LDH levels (Fig. 2a-d), we administered a single dose of Tocilizumab $(8 \mathrm{mg} / \mathrm{kg}, 360 \mathrm{mg})$ on day 3 of hospital admission (6 days from symptom onset). Fever immediately remitted and lymphocyte and monocyte counts, $\mathrm{CRP}$ and LDH progressively normalized (Fig. 2a-d); O2 therapy was discontinued 10 days after Tocilizumab with improvement of the atypical pneumonia (Fig. 2e-f).

Patient 3. A 70-year-old male on HD treatment for 12 years with undiagnosed CKD, diabetes mellitus, and dilated cardiomyopathy was admitted with fever and was found positive to SARS-CoV-2. He was treated with hydroxychloroquine ( $200 \mathrm{mg}$ two times/day for 2 days, then $200 \mathrm{mg} /$ post-HD for 10 days), ceftriaxone ( $1 \mathrm{~g} /$ day), prednisone (5 mg/day), enoxaparin (8000-10,000 UI/day) and oxygen at $8 \mathrm{lit} / \mathrm{min}$. After 4 days, due to clinical worsening he was treated with Tocilizumab $(8 \mathrm{mg} / \mathrm{kg} ; 640 \mathrm{mg})$. A rapid decrease in body temperature was observed and complete resolution of pneumonia was documented. 

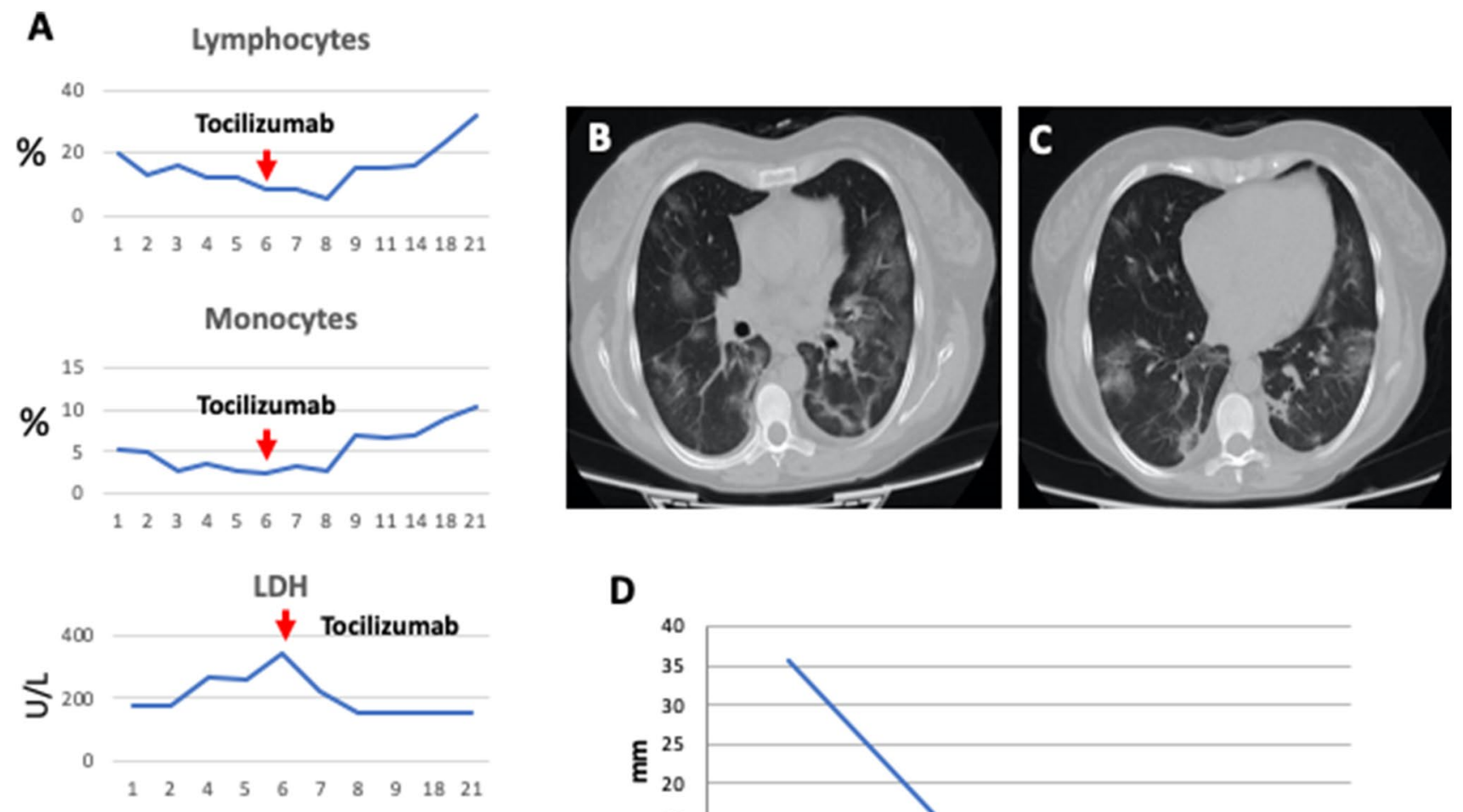

D
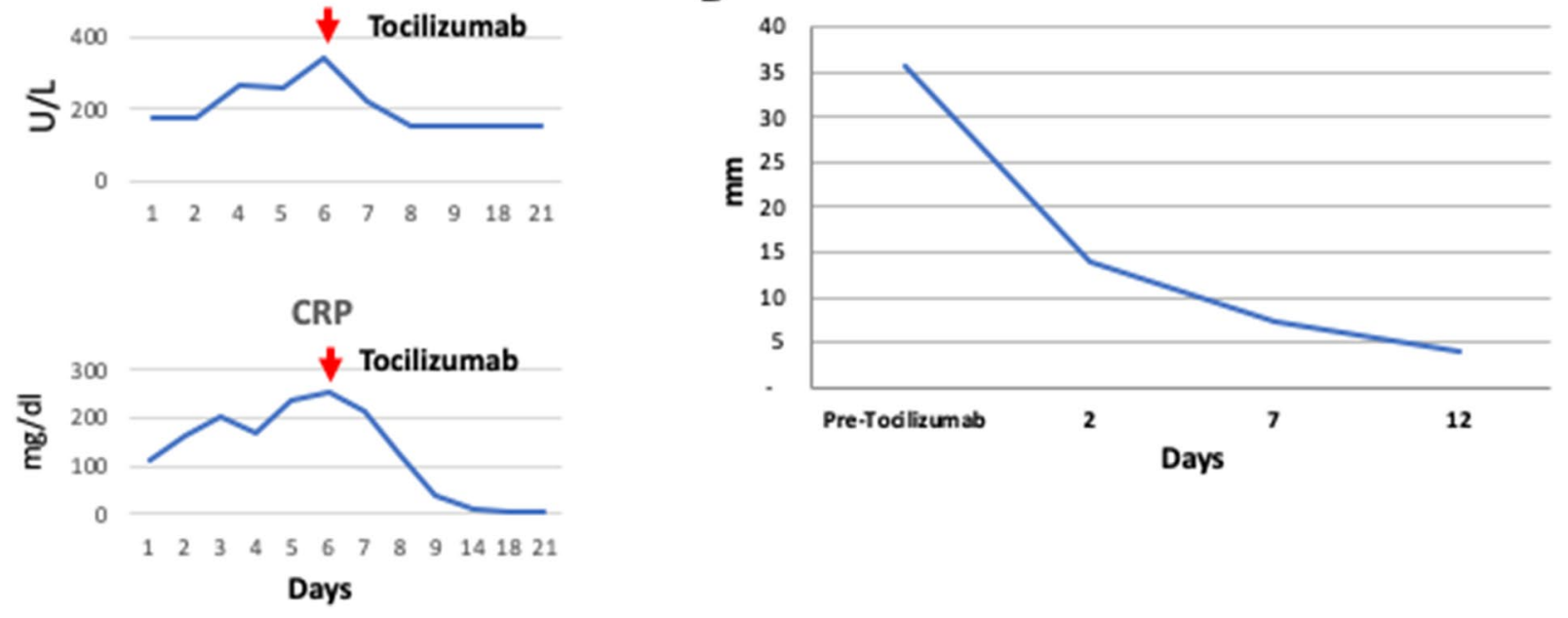

Fig. 1 Laboratory tests and chest manifestations before and after Tocilizumab administration in Patient 1. During the first days of hospital admission the patient presented a progressive and rapid decrease in Lymphocyte and Monocyte blood count, associated with a significant increase in LDH and CRP blood levels (a). The administration of Tocilizumab at day 6 resulted in a progressive improvement of all laboratory parameters (a). CT chest scan shows interstitial-alveolar multiple opacities with a 'ground-glass' look, some of which were

widely confluent, with a predominant mantle disposition and irregular triangular morphology, located, in particular, at the apex and dorsal segment of the upper left lobe, at the anterior and posterior segments of the upper right lobe, at the middle lobe, at the lingula site and at the lower lobes $(\mathbf{b}-\mathbf{c})$. Monitoring of lung lesions by chest echography: time course of lung lesion reduction after Tocilizumab administration $(\mathbf{d})$

\section{Lessons for the clinical nephrologists}

Frailty is defined as a state of increased vulnerability to stressful situations and has been correlated with advanced age and comorbidity [2]. Dialysis patients are characterized by an increased risk of cardiovascular, neoplastic, and infectious diseases, and are therefore more vulnerable to COVID-19. A recent report by the Italian Society of Nephrology showed that HD and peritoneal dialysis patients had a higher mortality rate in the COVID-19 pandemic (25 and $44 \%$ respectively) compared to the general population. This may be due to comorbidity, such as diabetes mellitus,

hypertension, dyslipidemia, and to non-traditional risk factors such as persistent inflammation generally defined as "dialysis syndrome".

COVID-19 is characterized by viral pneumonia as seen in our cases; dyspnea may occur 5-8 days after initial symptom onset, suggesting disease progression, evolving through acute respiratory distress syndrome (ARDS) and ultimately requiring intubation and mechanical ventilation [1]

Interestingly, our patients were not critically ill, but presented COVID-19 atypical pneumonia with a progressive alteration of biomarkers known to be associated with poor patient outcome [1]. Furthermore, patients were characterized by high interleukin-6 (IL-6) levels, a multi-functional 

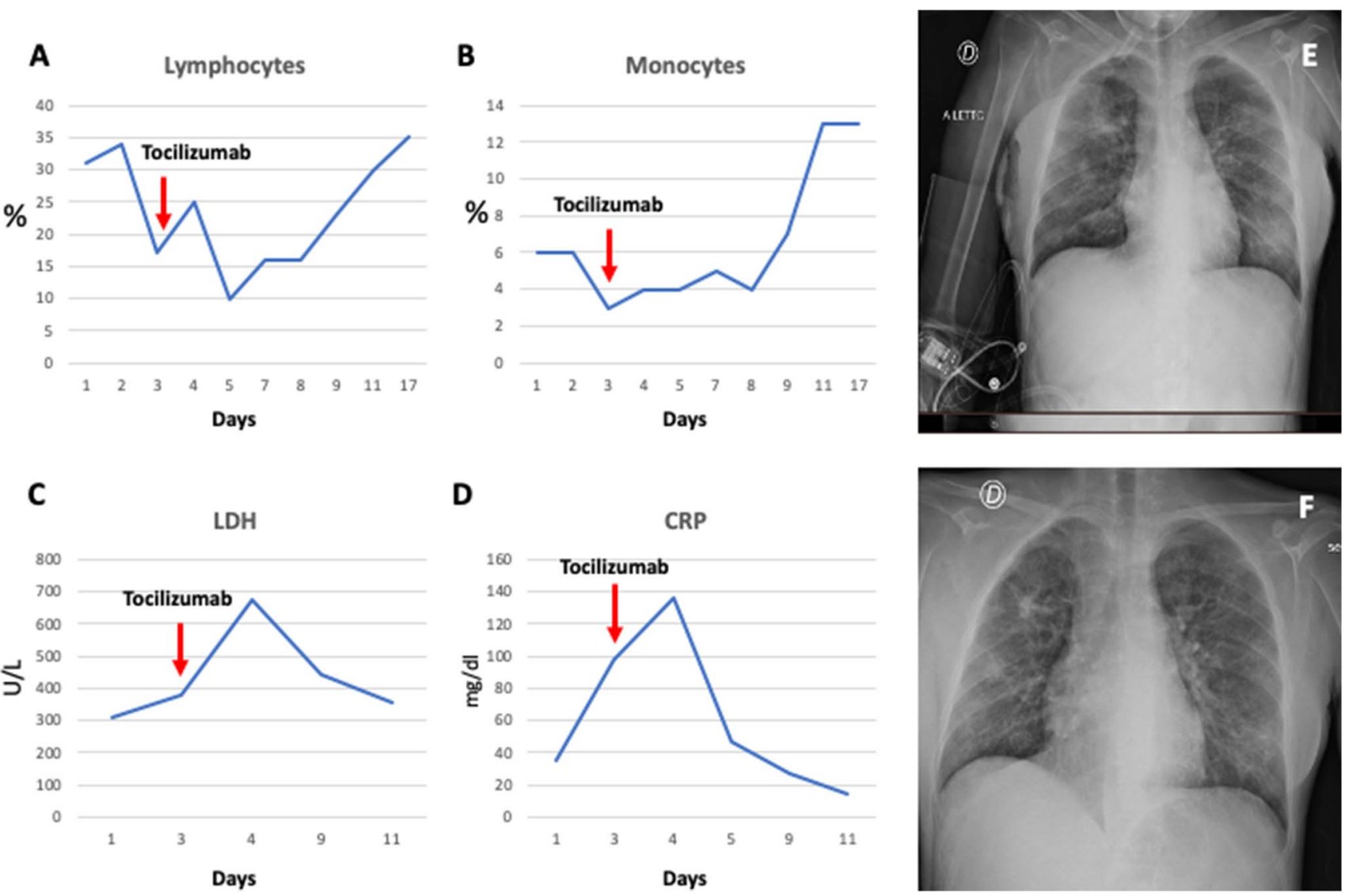

Fig. 2 Laboratory tests and chest manifestations before and after Tocilizumab administration in Patient 2. The administration of Tocilizumab at day 3 induced a progressive improvement of all the laboratory parameters. We found a progressive decrease in Lymphocyte (a) and Monocyte (b) blood count, associated with a significant increase

cytokine that promotes T-cell growth, activation and differentiation of B-cells and regulates the acute phase response in cases of systemic inflammation. This cytokine plays a pivotal role in ARDS, and high serum levels have been shown to correlate with mortality.

Given the extreme frailty of dialysis patients and considering the progressive deterioration of the clinical and laboratory parameters of our patients, we opted for early Tocilizumab administration [3] in an effort to avoid a sudden, precipitous deterioration in respiratory function that would have required immediate endotracheal intubation and mechanical ventilation.

Tocilizumab is a recombinant humanized anti-interleukin-6 receptor (IL-6R) monoclonal antibody that reduces IL-6 biological activity by interfering with the ability of IL-6 to bind the soluble and membrane-bound IL-6R and inhibit signal transduction. The use of Tocilizumab has been described in many reports, particularly in patients with established severe forms of COVID-19 pneumonia [4]; our approach in non-critically ill patients was very effective in LDH (c) and CRP blood levels (d). Evidence of new parenchymal thickening and bilateral widespread pulmonary interstitial involvement in pre-Tocilizumab administration (e); Bilateral improvement of lung lesions with a decrease in the density and in the extension of lung thicknesses as seen from X-ray images (f)

in reducing the clinical symptoms and altered laboratory parameters already after $24-48 \mathrm{~h}$ as seen in other clinical settings. In addition, this significant improvement was accompanied by a decrease in IL- 6 concentration. High IL- 6 serum levels before tocilizumab treatment suggest that the cytokine storm reaches a very high concentration in patients undergoing HD possibly because these frail patients with absent kidney function are unable to remove cytokines. This clinical feature of patients on HD and with COVID-19 infection may explain their higher mortality.

However, decision-making was based on published evidence which indicates that IL-6 levels increase in the early stages of COVID-19 pneumonia [5]. We hypothesized that an early IL-6 receptor blockade in our patients could prevent their progression towards ARDS. A recent case report described a patient on HD with COVID-19 who deteriorated quickly, developed ARDS and was treated with Tocilizumab at a later stage as compared to our patients, unfortunately no information on the patient's outcome or response to therapy was given [6]. In agreement with our data, early Tocilizumab 
administration at day 12 promoted a favorable outcome in a liver transplant recipient on HD [7]. Interestingly, Tocilizumab administration in some transplant patients led to a reduced requirement of $\mathrm{O} 2$ therapy and an improvement of lung lesions [4]. Conversely, other studies reported higher mortality in transplant patients treated with Tocilizumab [8]. Indeed, indiscriminate use of Tocilizumab should be avoided since it may induce profound immunosuppression and adverse events such as drug-induced liver injury, pancreatitis or pulmonary fibrosis [9].

In conclusion, we suggest that an approach based on early Tocilizumab administration could improve the outcome of COVID-19 atypical pneumonia in patients on hemodialysis, thereby protecting these frail patients.

Funding Not applicable.

\section{Compliance with ethical standards}

Conflicts of interest Not applicable.

Ethics approval Not applicable.

Consent to participate The patients gave consent for off-label use of Tocilizumab.

Consent for publication The patients gave consent for images or other clinical information related to this case report.

\section{References}

1. Li X, Xu S, Yu M et al (2020) Risk factors for severity and mortality in adult COVID-19 inpatients in Wuhan. J Allergy Clin Immunol. https://doi.org/10.1016/j.jaci.2020.04.006
2. Johansen KL, Chertow GM, Jin C, Kutner NG (2007) Significance of frailty among dialysis patients. J Am Soc Nephrol 18:29602967. https://doi.org/10.1681/ASN.2007020221

3. Gandhi RT, Lynch JB, Del Rio C (2020) Mild or Moderate Covid19. N Engl J Med. https://doi.org/10.1056/NEJMcp2009249

4. Xu X, Han M, Li T et al (2020) Effective treatment of severe COVID-19 patients with tocilizumab. Proc Natl Acad Sci USA. https://doi.org/10.1073/pnas.2005615117

5. Mehta P, McAuley DF, Brown M, et al (2020) COVID-19: consider cytokine storm syndromes and immunosuppression. Lancet 1033-1034

6. Ferrey AJ, Choi G, Hanna RM et al (2020) A case of novel coronavirus disease 19 in a chronic hemodialysis patient presenting with gastroenteritis and developing severe pulmonary disease. Am J Nephrol 51:337-342. https://doi.org/10.1159/000507417

7. Hammami MB, Garibaldi B, Shah P, et al (2020) Clinical course of COVID-19 in a liver transplant recipient on hemodialysis and response to tocilizumab therapy: a case report. Am J Transpl Off J Am Soc Transpl Am Soc Transpl Surg

8. Mella A, Mingozzi S, Gallo E, et al (2020) Case series of six kidney transplanted patients with COVID-19 pneumonia treated with tocilizumab. Transpl. Infect. Dis. e13348

9. Gatti M, Fusaroli M, Caraceni P et al (2020) Serious adverse events with tocilizumab: pharmacovigilance as an aid to prioritize monitoring in COVID-19. Br J Clin Pharmacol. https://doi. org/10.1111/bcp.14459

Publisher's Note Springer Nature remains neutral with regard to jurisdictional claims in published maps and institutional affiliations. 